\title{
The utility of point-of-care ultrasound in the assessment of volume status in acute and critically ill patients
}

\author{
Ali Pourmand, Matthew Pyle, David Yamane, Kazi Sumon, Sarah E. Frasure \\ Department of Emergency Medicine, George Washington University School of Medicine and Health Sciences, Washington, \\ $D C, U S A$
}

Corresponding Author: Sarah E. Frasure, Email: sarahfrasure@yahoo.com

BACKGROUND: Volume resuscitation has only been demonstrated to be effective in approximately fifty percent of patients. The remaining patients do not respond to volume resuscitation and may even develop adverse outcomes (such as acute pulmonary edema necessitating endotracheal intubation). We believe that point-of-care ultrasound is an excellent modality by which to adequately predict which patients may benefit from volume resuscitation.

DATA RESOURCES: We performed a search using PubMed, Scopus, and MEDLINE. The following search terms were used: fluid responsiveness, ultrasound, non-invasive, hemodynamic, fluid challenge, and passive leg raise. Preference was given to clinical trials and review articles that were most relevant to the topic of assessing a patient's cardiovascular ability to respond to intravenous fluid administration using ultrasound.

RESULTS: Point-of-care ultrasound can be easily employed to measure the diameter and collapsibility of various large vessels including the inferior vena cava, common carotid artery, subclavian vein, internal jugular vein, and femoral vein. Such parameters are closely related to dynamic measures of fluid responsiveness and can be used by providers to help guide fluid resuscitation in critically ill patients.

CONCLUSION: Ultrasound in combination with passive leg raise is a non-invasive, costand time-effective modality that can be employed to assess volume status and response to fluid resuscitation. Traditionally sonographic studies have focused on the evaluation of large veins such as the inferior vena cava, and internal jugular vein. A number of recently published studies also demonstrate the usefulness of evaluating large arteries to predict volume status.

KEY WORDS: Stroke volume; Fluid responsiveness; Ultrasound; Non-invasive; Hemodynamic

\section{INTRODUCTION}

Point-of-care ultrasound provideplrs a non-invasive method for clinicians to evaluate the intravascular volume status and fluid responsiveness of a patient. ${ }^{[1]}$ This tool allows the clinician to rapidly make critical decisions regarding appropriate treatment of critically-ill patients (volume resuscitation versus the use of vasopressors). ${ }^{[2]}$ Predicting fluid responsiveness accurately also prevents volume overload which can lead to adverse events such as acute pulmonary edema.

Previously, dynamic measures such as variations in stroke volume and pulse pressure were used to assess fluid responsiveness. ${ }^{[3]}$ Yet there are risks in obtaining and limitations of interpretation of these measurements, which preclude them from being practical in most patients. ${ }^{[3]}$ In contrast, expeditious sonographic evaluation of the major vessels at the bedside of a critically ill patient provides a highly sensitive and specific assessment of intravascular volume status and response to fluid administration. ${ }^{[4]}$

Furthermore, the use of point-of-care ultrasound to evaluate vessel diameter and collapsibility in 
conjunction with a passive leg raise (PLR) as a form of fluid challenge offers a valuable method to predict fluid responsiveness. ${ }^{[5]}$ The following article is a discussion of ongoing progress in accurate fluid assessment of critically ill patients.

\section{METHODS}

We performed a literature review using PubMed, Scopus, and MEDLINE databases in order to evaluate the current literature on volume status assessment in critically ill patients. The following search terms were applied: fluid responsiveness, ultrasound, hemodynamic, non-invasive, fluid challenge, and PLR. Two senior authors also reviewed the bibliographies of each article to identify additional studies. Exclusionary criteria included studies that were not published in English, abstracts, unpublished data, animal studies, and duplicate articles across multiple search criteria. Preference was given to clinical trials and review articles that were most relevant to the evaluation of a patient's cardiovascular capacity to respond to intravenous fluid administration with pointof-care ultrasound.

\section{RESULTS}

\section{Traditional assessment of fluid responsiveness}

Conventionally, clinicians treat critically ill patients with judicious administration of intravenous crystalloid fluids. ${ }^{[6]}$ Other resuscitative techniques include intravenous infusions of blood products and colloids as well as pharmacological manipulation of physiology with the use of vasopressors. Each of these methods carries risk. For example, colloids, such as albumin, remain sequestered in the intravascular space for long periods of time, yet they are prohibitively expensive. Furthermore, research has failed to demonstrate a mortality benefit of colloids when compared to crystalloid administration. ${ }^{[6,7]}$

Predicting fluid responsiveness is akin to determining a patient's position on the Frank-Starling curve. Patients will respond to fluid administration if they are on the ascending portion of the Starling curve. Critically ill patients may, however, exist at the plateau of the curve in which case an increase in preload no longer confers an advantage.$^{[6]}$ Since the shape of the Starling curve varies between patients it can be very challenging to determine a patient's position on the curve ${ }^{[6]}$ In order to determine appropriate treatment of critically ill patients the provider must be able to assess both intravascular volume status and fluid responsiveness. Traditionally, clinical measures to assess these states have included static measures, such as central venous pressure (CVP) and pulmonary artery occlusion pressure, and dynamic measures, such as variations in stroke volume and pulse pressure. ${ }^{[3]}$

Static measures (CVP and pulmonary artery occlusion pressure) characterize preload and can theoretically determine a patient's position on the Starling curve. Yet the shape of the Starling curve differs significantly between individuals. Not surprisingly, therefore, static measures have proven unable to accurately determine fluid responsiveness. ${ }^{[5-12]}$ A metaanalysis of 23 research studies demonstrated that invasive monitoring of CVP was not only a poor predictor of fluid responsiveness, but also conferred numerous risks including infection and mechanical complications. ${ }^{[6]}$ The measurement of pulmonary artery occlusion pressure requires the placement of a pulmonary artery catheter; this device is also associated with increased morbidity and mortality. ${ }^{[13]}$

Dynamic calculations of stroke volume and pulse pressure have consistently demonstrated reliable predictions of the degree of fluid responsiveness. ${ }^{[3,6,14]}$ However, variations in pulse pressure are determined by measuring intrathoracic pressure changes during mechanical ventilation. Even minimal spontaneous breathing by a patient who is mechanically ventilated can alter intrathoracic measurements. Therefore, mechanically ventilated patients must be heavily sedated for pulse pressure variations to have predictive value. ${ }^{[3,10,15]}$ It is not possible to calculate accurate variations in pulse pressure in patients who are not undergoing such interventions, limiting the applicability of this method ${ }^{[6]}$ Pulse pressure variations are also not predictive of fluid responsiveness in patients with arrhythmias, low tidal volumes, a low ratio between cardiac and respiratory cycles, or those with an open chest. ${ }^{[3,10]}$

\section{Understanding fluid challenge and therapy}

A fluid challenge is an administration of a small bolus of intravenous fluid to assess a patient's hemodynamic response. Providing a small bolus of fluid followed by clinician reassessment reduces the risk of acute pulmonary edema. ${ }^{[7]}$ In the past CVP and pulmonary artery occlusion pressure were measured before and in ten-minute intervals after a fluid challenge in order to predict fluid responsiveness. The numerical change in pressure would determine whether or not the infusion could be safely continued ${ }^{[7,8]}$ New research, however, has examined the use of a PLR to determine fluid responsiveness. ${ }^{[9]}$ 


\section{The utility of passive leg-raising}

To perform a PLR the provider lifts the legs of a patient from a horizontal supine position. This technique induces a gravitational mobilization of intravascular volume from the lower extremities towards the heart. ${ }^{[8]}$ The maneuver requires the operator to lift the patient's legs from zero to forty-five degrees while measuring stroke volume and cardiac output before and after the maneuver. ${ }^{[16]}$ PLR redistributes $300-500 \mathrm{~mL}$ of intravascular volume to the heart. PLR is, thus, also referred to as an "autotransfusion". This method is especially useful because it is quickly reversible. PLR carries no risk of fluid overload and is generally assessed via the change in stroke volume using point-of-care ultrasound. $^{[9]}$

Passive leg-raising has been proposed as a way to assess fluid responsiveness because most patients, especially in the early phases of resuscitation, are not intubated, and do not meet the criteria for the use of invasive measures of fluid responsiveness. ${ }^{[8]}$ Several prospective cohort studies have demonstrated that an increase in stroke volume over ten percent, as induced by PLR, was predictive of fluid responsiveness, with a sensitivity of $77 \%$ to $100 \%$, and a specificity of $88 \%$ and $99 \%{ }^{[4,17,18]}$ A prospective cohort study in 2012 by Monnet et al ${ }^{[19]}$ demonstrated that pulse pressure variation (a dynamic measure) predicted fluid responders with equivalent accuracy to PLR in patients with greater respiratory compliance. However, the effectiveness of pulse pressure variation was much worse when compared to PLR in patients with lower respiratory system compliance. In summary, PLR is an easily performed, non-invasive way to assess volume status.

\section{Assessment through echocardiography}

Echocardiography is a non-invasive tool which allows the provider to evaluate changes in stroke volume and possesses a lower risk profile compared to other methods, including pulmonary artery catheterization. ${ }^{[16]}$ Furthermore, point-of-care cardiac ultrasound can be quickly performed at the bedside by the clinician and may be repeated as the clinical situation changes. Transthoracic echocardiography (TTE) is generally preferred over transesophageal echocardiography (TEE) in emergent situations, although TEE may be useful in intubated or sedated patients. ${ }^{[1,16]}$

The clinician can also use point-of-care ultrasound to assess for changes in the inferior vena cava (IVC) diameter throughout the respiratory cycle in order to determine fluid responsiveness. Because the diameter of the left ventricular outflow tract is assumed to be static throughout the respiratory and cardiac cycle, any change in aortic blood flow should represent a change in stroke volume. Clinicians with advanced skills in transthoracic echocardiography can also obtain a Doppler measurement of the maximal ascending aortic flow velocity (just distal to the aortic valve) immediately before and after performing a PLR. ${ }^{[16]}$ Variations in aortic flow velocity throughout the respiratory cycle accurately predict fluid responsiveness. A variation of greater than $14 \%$ has a high positive predictive value for the patient being fluid responsive while values less than $10 \%$ are associated with a high negative predictive value. ${ }^{[20]}$ When performing a TTE the maximal ascending aortic flow velocity can best be obtained in the apical five-chamber view; the deep gastric view is most conducive when using TEE. ${ }^{[16]}$

A systematic review in 2011 by Mandeville and Colebourn demonstrated that TTE is an excellent modality with which to measure stroke volume and cardiac output in order to predict and determine volume responsiveness in hemodynamically unstable patients. ${ }^{[4]}$ Of note, TTE is also accurate when performed on patients who are spontaneously breathing and/or have existing cardiac arrhythmias. In contrast, the volume responsiveness of such patients cannot be reliably determined with traditional forms of assessment such as arterial waveforms.

The clinician should be aware of limitations associated with ultrasound. Obese body habitus, prominent ribs, emphysematous lungs, subcutaneous emphysema, surgical dressings, an open chest in the post cardiac surgery patient, life-saving devices (such as defibrillation pads), or chest wounds, may significantly degrade sonographic images. ${ }^{[1,4]}$

\section{Advantages and utility of ultrasound}

The ideal test to evaluate the hemodynamic status of a patient would be easy to use, cheap, noninvasive, produce clinically useful information, and enjoy low inter-operator variability. ${ }^{[21]}$ Ultrasound has been embraced by providers performing procedures (ultrasound-guided peripheral and central lines, paracentesis, thoracentesis, pericardiocentesis, abscess incision and drainage etc). In the emergency department point-of-care ultrasound is also a vital component of trauma examinations, and can expedite work-ups of patients with pulmonary, cardiovascular, obstetric, musculoskeletal, and abdominal complaints. ${ }^{[21]}$ Moreover, ultrasound is emerging as an essential tool 
to guide interventions for hemodynamically unstable patients. Although the field of functional hemodynamics is in its infancy, it has become evident that assessing changes in IVC diameter over the period of the respiratory cycle offers more insight into a patient's volume status than static measures can. ${ }^{[1]}$ Ultrasound also assesses the venous collapsibility index, which is a measurement of small changes in the diameters of large veins throughout the respiratory cycle. ${ }^{[21]}$ The collapsibility index is determined by the following formula:

The collapsibility index $=($ maximal vessel diameter minimal vessel diameter $) \div$ maximal vessel diameter.

By using multiple vessel diameters, the venous collapsibility index provides an internal patient-specific standard ${ }^{[21]}$ It has also been demonstrated that venous collapsibility may be inversely proportional to CVP: a $1 \mathrm{mmHg}$ change in central venous pressure correlates to about $3.3 \%$ change in IVC collapsibility. ${ }^{[1]}$

\section{Understanding measurement of IVC (diameter, collapsibility, inspiration, and expiration) in relation to CVP}

A study in 2016 by Karacabey et $\mathrm{a}^{[2]}$ revealed that measures of IVC collapsibility were inversely correlated with CVP measurements in intubated patients. If the diameter of the IVC is less than $2.1 \mathrm{~cm}$ and decreases by more than half during inhalation, then the right atrial pressure sits within the normal range of $0-5 \mathrm{mmHg}$. If the IVC has a diameter of greater than $2.1 \mathrm{~cm}$ and decreases by less than half with inspiration, the pressure in the right atrium is between $10-20 \mathrm{mmHg}$. Ilyas et $\mathrm{al}^{[22]}$ noted that CVP is proportional to IVC diameter but inversely proportional to the IVC collapsibility index (CI). Although it has not been demonstrated that CVP and $\mathrm{CI}$ are linearly related, the closer the $\mathrm{CI}$ is to either $0 \%$ or $100 \%$, the more likely it is that the patient is volume depleted or volume-overloaded respectively.

\section{Understanding measurement of IVC (diameter and collapsibility) in relation to measurements of internal jugular vein and femoral vein}

The relationship between IVC collapsibility and internal jugular vein (IJV) collapsibility is still unclear. ${ }^{[23]}$ There exists a correlation between the collapsibility of the IVC and the IJV, yet this correlation is heavily dependent on intra-thoracic and intraabdominal pressures at the time of measurement. ${ }^{[24]}$ IJV collapsibility index values over $39 \%$ are strongly indicative of volume depletion. A study in 2015 by Bauman et $\mathrm{al}^{[24]}$ showed that increased intra-abdominal pressure can cause collapse of the IJV, which could be falsely interpreted as volume depletion. Compared to other large veins in the body the IVC still stands as the most evidence-based indicator of volume status.

\section{Interrater reliability of IVC assessment}

Although point-of-care ultrasound has largely been adopted for IVC assessment, image interpretation requires provider training and experience. Bowel gas and large body habitus significantly impact image acquisition and interpretation. Accurate measurement of the IVC is also subject to the cylinder-tangent effect; measurements in the long axis which are closer to the walls will be falsely lower than measurements in the true center of the vessel. Existing literature notes that discrepancies in IVC diameter measurements can exist between users, particularly when comparing experts with novice sonographers. ${ }^{[25]}$ However, a study by Akkaya et $\mathrm{al}^{[26]}$ in 2013 demonstrated moderate reliability of IVC measurement between emergency medicine residents of different post-graduate years.

\section{Assessment of corrected flow time}

Corrected flow time is a measurement of systolic flow time that is corrected for heart rate and can be determined using pulsed wave spectral Doppler. ${ }^{[27]}$ It is a non-invasive way to determine a patient's intravascular volume using point-of care ultrasound. Pare et $\mathrm{al}^{[27]}$ concluded that corrected flow time accurately predicted decrease in preload after the administration of nitroglycerin. In addition, Hossein-Nejad et al ${ }^{[28]}$ noted that corrected flow time can be determined via electrocardiogram when assessing a patient's volume status. Corrected flow time measurements were comparable between Doppler evaluation and electrocardiogram. ${ }^{[28,29]}$

\section{Measurements related to the common carotid artery (diameter, systolic flow time)}

Given the emphasis on the visualization of large veins in assessing a patient's response to intravenous fluids, changes in arterial vessels have largely been overlooked. ${ }^{[30]}$ The diameter of the common carotid artery increases with intravascular volume expansion, and is also correlated with the change in pulse pressure variation. Providers can also use point-of care ultrasound to evaluate carotid systolic flow time. ${ }^{[31]}$ An increase of carotid systolic flow time of greater than thirty milliseconds after PLR was found to correspond to changes in the volume status of patients who had completed a dialysis session. 


\section{Understanding correct flow time within common carotid artery}

Corrected flow time can also be applied to arterial measurements. When intravenous fluids were administered to volume-depleted patients, researchers detected changes in the carotid artery corrected flow time using ultrasound with Doppler before a change in the patient's vital signs became apparent. ${ }^{[32]}$ Similarly, carotid artery corrected flow time as evaluated with ultrasound accurately reflected delivery of lower extremity intravascular volume to the heart after PLR in healthy volunteers. ${ }^{[28]}$

\section{Detecting fluid overload with ultrasound}

In addition to predicting fluid responsiveness, ultrasound can help providers to look for volume overload in the form of pulmonary edema. Fluid overload triggers increased density of the interlobular septae of the lungs, creating hyper-echoic reverberation artifacts at the pleura, known as B-lines. ${ }^{[33]} \mathrm{B}$-lines can be identified in sub-clinical overload; their number increases proportionately to the degree of pulmonary edema. ${ }^{[34]}$ Of note, sonographic detection of pulmonary congestion is also more sensitive than chest X-ray or auscultation. ${ }^{[35]}$

\section{Application of intravascular volume measurement - special cases}

Other research has focused on the use of ultrasound to assess the volume status of pregnant patients, intubated patients, patients in shock, and patients with congestive heart failure. A prospective observational study examined the IVC diameter in pregnant women before and after an intravenous fluid bolus and determined that changes in diameter could be reliably assessed. ${ }^{[36]}$ Another important population includes mechanically ventilated patients. The use of general anesthetics is associated with hypotension, which may lead to adverse outcomes. ${ }^{[37]}$ Ultrasound can predict post-anesthesia hypotension by measuring the IVC CI and diameter

A recent study $^{[38]}$ has also evaluated the utility of ultrasound in detecting volume status in the pre-hospital setting. Ultrasound showed that subjects with acute mountain sickness have greater intravascular volume than controls. ${ }^{[38]}$ Thus, patients with acute mountain sickness seem to retain fluid in an altitude-dependent manner. Ultrasound can be used to reliably detect increases in volume following successful resuscitation. ${ }^{[39-42]}$ Finally, patients with acute decompensated heart failure require strict intravascular fluid monitoring. Krishnan et $\mathrm{al}^{[43]}$ showed that providers could use ultrasound evaluation of the IVC diameter to predict a patient's response to diuretics independent of his/her baseline renal function.

\section{DISCUSSION}

Ultrasound has become an increasingly important assessment of volume status and response to volume resuscitation in critically ill patients. Besides being non-invasive and low in cost, the clinician can repeat the ultrasound at the patient's bedside as often as needed. ${ }^{[1,21]}$ Much research has focused on the elasticity of large veins, such as the inferior vena cava and the internal jugular vein, to calculate standardized parameters for the determination of fluid responsiveness, including the collapsibility index..$^{[2,22-24]}$ These parameters are related to more traditional predictors of fluid responsiveness like central venous pressure. Emerging studies, however, demonstrate that large arteries, such as the common carotid artery, are also dynamically compliant, and can predict fluid responsiveness. ${ }^{[30-32]}$

Certainly, ultrasound is operator dependent. ${ }^{[25,26]}$ Standardized parameters, such as corrected flow time, can be calculated using ultrasound, which may help to eliminate differences in image interpretation between providers. ${ }^{[27]}$ Ultrasound can also assess whether a patient undergoing volume resuscitation is developing pulmonary congestion. Ultrasound is more sensitive than plain film imaging or auscultation when looking for pulmonary edema.

\section{Limitations}

There are several limitations to this review article. We included a variety of studies that differ in many ways and are linked only by subject area. Despite the heterogeneity of sample size and type of patients in the studies that we examined, however, they were all valued with the same significance. Given that the studies mostly support one another, the fallacy of comparing articles with different strengths is not as grave a fault. Certainly, the amount and type of intravenous fluid, as well as the rate of fluid administration are not unified across the literature. Finally, ultrasound is operator dependent. More experienced clinicians are more facile with this tool, and consequently more likely to make the proper diagnosis based on accurate image interpretation, than the novice sonographer.

\section{CONCLUSION}

In conclusion, point-of-care ultrasound, in 
combination with PLR, is a non-invasive, low cost, and time-effective modality that can be employed by a provider to examine volume status and predict patient response to fluid resuscitation. Traditionally, research studies have focused on the evaluation of large veins, but a few studies now demonstrate that large arteries may provide similar insight into a patient's volume status. As future studies further expand our understanding of the accuracy of sonographic measurements to examine volume status, the use of point-of-care ultrasound in the assessment of a critically ill patient will hopefully become the standard of care.

Funding: This research received no specific grant from any funding agency, commercial or not-for-profit sectors.

Ethical approval: Not needed.

Conflicts of interest: Authors have no financial or other conflicts of interest related to this submission.

Contributors: AP proposed the study and wrote the first draft. All authors read and approved the final version of the paper.

\section{REFERENCES}

1 Kelly N, Esteve R, Papadimos TJ, Sharpe RP, Keeney SA, DeQuevedo R, et al. Clinician-performed ultrasound in hemodynamic and cardiac assessment: a synopsis of current indications and limitations. Eur J Trauma Emerg Surg. 2015;41(5):469-80.

2 Karacabey S, Sanri E, Guneysel O. A non-invasive method for assessment of intravascular fluid status: Inferior vena cava diameters and collapsibility index. Pak J Med Sci. 2016;32(4):836-40.

3 Ansari BM, Zochios V, Falter F, Klein AA. Physiological controversies and methods used to determine fluid responsiveness: A qualitative systematic review. Anaesthesia. 2016;71(1):94-105.

4 Mandeville JC, Colebourn CL. Can transthoracic echocardiography be used to predict fluid responsiveness in the critically ill patient? A systematic review. Crit Care Res Pract. 2012;2012:513480.

5 Marik PE, Lemson J. Fluid responsiveness: An evolution of our understanding. Br J Anaesth. 2014;112(4):617-20.

6 Mackenzie DC, Noble VE. Assessing volume status and fluid responsiveness in the emergency department. Clin Exp Emerg Med. 2014;1(2):67-77.

7 Vincent JL, Weil MH. Fluid challenge revisited. Crit Care Med. 2006;34(5):1333-7.

8 Mark PE, Monnet X, Teboul JL. Hemodynamic parameters to guide fluid therapy. Ann Intensive Care. 2011;1(1):1.

9 Arumugam SK, Mudali I, Strandvik G, El-Menyar A, Al-Hassani A, Al-Thani H. Risk factors for ventilator-associated pneumonia in trauma patients: A descriptive analysis. World J Emerg Med. 2018;9(3):203-10.

10 Monnet X, Teboul JL. Assessment of volume responsiveness during mechanical ventilation: Recent advances. Crit Care. 2013;17(2):217.
11 Teboul JL, Monnet X. Prediction of volume responsiveness in critically ill patients with spontaneous breathing activity. Curr Opin Crit Care. 2008;14(3):334-9.

12 Cheung WYS, Cheung WK, Lam CH, Chan YW, Chow HC, Cheng KL, et al. Intravenous fluid selection rationales in acute clinical management. World J Emerg Med. 2018;9(1):13-9.

13 Connors AF, Jr Speroff T, Dawson NV, Thomas C, Harrell FE Jr, Wagner $\mathrm{D}$, et al. The effectiveness of right heart catheterization in the initial care of critically ill patients. JAMA. 1996;276(11):88997.

14 Marik PE, Cavallazzi R, Vasu T, Hirani A. Dynamic changes in arterial waveform derived variables and fluid responsiveness in mechanically ventilated patients: A systematic review of the literature. Crit Care Med. 2009;37(9):2642-7.

15 Cannesson M, Le Manach Y, Hofer CK, Goarin JP, Lehot JJ, Vallet B, et al. Assessing the diagnostic accuracy of pulse pressure variations for the prediction of fluid responsiveness: a "gray zone" approach. Anesthesiology. 2011;115(2):231-41.

16 Levitov A, Marik PE. Echocardiographic assessment of preload responsiveness in critically ill patients. Cardiol Res Pract. 2012;2012:819696.

17 Monnet X, Rienzo M, Osman D, Anguel N, Richard C, Pinsky $\mathrm{MR}$, et al. Passive leg raising predicts fluid responsiveness in the critically ill. Crit Care Med. 2006;34(5):1402-7.

18 Préau S, Saulnier F, Dewavrin F, Durocher A, Chagnon JL. Passive leg raising is predictive of fluid responsiveness in spontaneously breathing patients with severe sepsis or acute pancreatitis. Crit Care Med. 2010;38(3):819-25.

19 Monnet X, Bleibtreu A, Ferré A, Dres M, Gharbi R, Richard $\mathrm{C}$, et al. Passive leg-raising and end-expiratory occlusion tests perform better than pulse pressure variation in patients with low respiratory system compliance. Crit Care Med. 2012;40(1):1527.

20 Feissel M, Michard F, Mangin I, Ruyer O, Faller JP, Teboul JL. Respiratory changes in aortic blood velocity as an indicator of fluid responsiveness in ventilated patients with septic shock. Chest. 2001;119(3):867-73.

21 Finnerty NM, Panchal AR, Boulger C, Vira A, Bischof JJ, Amick $\mathrm{C}$, et al. Inferior vena cava measurement with ultrasound: what is the best view and best mode? West $\mathrm{J}$ Emerg Med. 2017;18(3):496-501.

22 Ilyas A, Ishtiaq W, Assad S, Ghazanfar H, Mansoor S, Haris $\mathrm{M}$, et al. Correlation of IVC diameter and collapsibility index with central venous pressure in the assessment of intravascular volume in critically ill patients. Cureus. 2017;9(2):e1025.

23 Kent A, Patil P, Davila V, Bailey JK, Jones C, Evans DC, et al. Sonographic evaluation of intravascular volume status: Can internal jugular or femoral vein collapsibility be used in the absence of IVC visualization? Ann Thorac Med. 2015;10(1):449.

24 Bauman Z, Coba V, Gassner M, Amponsah D, Gallien J, Blyden $\mathrm{D}$, et al. Inferior vena cava collapsibility loses correlation with internal jugular vein collapsibility during increased thoracic or intra-abdominal pressure. J Ultrasound. 2015;18(4):343-8.

25 Bowra J, Uwagboe V, Goudie A, Reid C, Gillett M. Interrater agreement between expert and novice in measuring inferior vena cava diameter and collapsibility index. Emerg Med Australas. 2015;27(4):295-9.

26 Akkaya A, Yesilaras M, Aksay E, Sever M, Atilla OD. The interrater reliability of ultrasound imaging of the inferior vena cava performed by emergency residents. Am J Emerg Med. 
2013;31(10):1509-11.

27 Pare JR, Liu R, Moore CL, Safdar B. Corrected flow time: a noninvasive ultrasound measure to detect preload reduction by nitroglycerin. Am J Emerg Med. 2016;34(9):1859-62.

28 Hossein-Nejad H, Banaie M, Davarani SS, Mohammadinejad P. Assessment of corrected flow time in carotid artery via pointof-care ultrasonography: Reference values and the influential factors. J Crit Care. 2017;(40):46-51.

29 Hossein-Nejad H, Mohammadinejad P, Zeinoddini A, Seyedhosseini Davarani S, Banaie M. A new modality for the estimation of corrected flow time via electrocardiography as an alternative to Doppler ultrasonography. Ann Noninvasive Electrocardiol. 2018;23(1):1-7.

30 Hilbert T, Klaschik S, Ellerkmann RK, Putensen C, Thudium M. Common carotid artery diameter responds to intravenous volume expansion: an ultrasound observation. Springerplus. 2016;5(1):853.

31 Antiperovitch P, Iliescu E, Chan B. Carotid systolic flow time with passive leg raise correlates with fluid status changes in patients undergoing dialysis. J Crit Care. 2017;(39):83-6.

32 Blehar DJ, Glazier S, Gaspari RJ. Correlation of corrected flow time in the carotid artery with changes in intravascular volume status. J Crit Care. 2014;29(4):486-8.

33 Picano E, Frassi F, Agricola E, Gligorova S, Gargani L, Mottola G. Ultrasound lung comets: A clinically useful sign of extravascular lung water. J Am Soc Echocardiogr. 2006;19(3):356-63.

34 Agricola E, Bove T, Oppizzi M, Marino G, Zangrillo A, Margonato A, et al. "Ultrasound comet-tail images": A marker of pulmonary edema - A comparative study with wedge pressure and extravascular lung water. Chest. 2005;127(5):1690-5.

35 Torino C, Gargani L, Sicari R, Letachowicz K, Ekart R, Fliser D, et al. The agreement between auscultation and lung ultrasound in hemodialysis patients: The LUST study. Clin J Am Soc Nephrol. 2016;11(11):2005-11.

36 Hernandez CA, Reed KL, Juneman EB, Cohen WR. Changes in sonographically measured inferior vena caval diameter in response to fluid loading in term pregnancy. J Ultrasound Med. 2016;35(2):389-94.

37 Zhang J, Critchley H. Inferior vena cava ultrasonography before general anesthesia can predict hypotension after induction. Anesthesiology. 2016;123(3):580-9.

38 Pitman JT, Thapa GB, Harris NS. Field ultrasound evaluation of central volume status and acute mountain sickness. Wilderness Environ Med. 2015;26(3):319-26.

39 Zhang X, Luan H, Zhu P, Feng J, Cui J, Zhao Z. Does ultrasonographic measurement of the inferior vena cava diameter correlate with central venous pressure in the assessment of intravascular volume in patients undergoing gastrointestinal surgery? J Surg Res. 2014;191(2):339-43.

40 Zhu P, Zhang X, Luan H, Feng J, Cui J, Wu Y, et al. Ultrasonographic measurement of the subclavian vein diameter for assessment of intravascular volume status in patients undergoing gastrointestinal surgery: Comparison with central venous pressure. J Surg Res. 2015;196(1):102-6.

41 Huggins JT, Doelken P, Walters C, Rockey DC. Point-ofcare echocardiography improves assessment of volume status in cirrhosis and hepatorenal syndrome. Am J Med Sci. 2016;351(5):550-3.

42 Chambers KA, Park AY, Banuelos RC, Darger BF, Akkanti BH, Macaluso A, et al. Outcomes of severe sepsis and septic shock patients after stratification by initial lactate value. World J Emerg Med. 2018;9(2):113-7.

43 Krishnan DK, Pawlaczyk B, McCullough PA, Enright S, Kunadi A, Vanhecke TE. Point-of-care, ultraportable echocardiography predicts diuretic response in patients admitted with acute decompensated heart failure. Clin Med Insights Cardiol. 2016;10:201-8.

Received April 9, 2019 Accepted after revision July 10, 2019 